\title{
Impact of laparoscopic antireflux surgery on belching in pediatric GERD patients
}

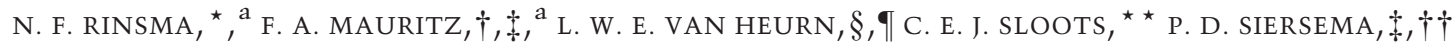 \\ R. H. J. HOUWEN, + † D. C. VAN DER ZEE, † A. A. M. MASCLEE, ${ }^{\star}$ J. M. CONCHILLO * \& \\ M. Y. A. VAN HERWAARDEN-LINDEBOOM†
}

\begin{abstract}
*Department of Gastroenterology and Hepatology, Maastricht University Medical Center, Maastricht, The Netherlands $\dagger$ Department of Pediatric Surgery, Wilhelmina Children's Hospital, Utrecht, The Netherlands

†Department of Gastroenterology and Hepatology, University Medical Center Utrecht, Utrecht, The Netherlands $\S$ Department of Pediatric Surgery, Maastricht University Medical Center, Maastricht, The Netherlands

qDepartment of Pediatric Surgery, Emma Children's Hospital, Amsterdam Medical Center, Amsterdam, The Netherlands.

**Department of Pediatric Surgery, Erasmus MC - Sophia Children's Hospital, Rotterdam, The Netherlands

$\dagger$ †Department of Gastroenterology and Hepatology, Radboud University Medical Center, Nijmegen, The Netherlands

†tDepartment of Pediatric Gastroenterology and Hepatology, Wilhelmina Children's Hospital, Utrecht, The Netherlands
\end{abstract}

\section{Key Points}

- Laparoscopic antireflux surgery (LARS) is associated with gas-related complications that may be due to the inability to belch. Objective data on the effect of LARS on belching are currently not available in children with GERD.

- LARS significantly reduced the number of gastric belches (GBs), whereas the number of air swallows and supragastric belches (SGBs) remains unchanged.

- The reduction in gastric belching is likely to explain frequently reported postoperative gas-related complications of LARS in children.

\begin{abstract}
Background Laparoscopic antireflux surgery (LARS) is a well-established treatment option for children with proton pomp inhibitor (PPI)-resistant gastroesophageal reflux disease (GERD). Besides preventing reflux of gastric fluid and solid content, LARS may also impair the ability of the stomach to vent intragastric air (i.e. gastric belching) and induce gas-related complications, such as bloating and/or hyperflatulence. Furthermore, it was previously hypothesized that LARS induces a behavioral type of belching, not originating from the stomach, called supragastric
\end{abstract}

\section{Address for Correspondence}

Nicolaas F. Rinsma, Department of Gastroenterology and Hepatology, Maastricht University Medical Center, Postbox 5800, 6202 AZ Maastricht, The Netherlands.

Tel: +31 43 3875021; fax: +31 43 3875006;

e-mail: n.rinsma@maastrichtuniversity.nl

${ }^{a}$ Both authors contributed equally to this study work.

Received: 14 February 2016

Accepted for publication: 11 April 2016 belching. The aim of this study was to objectively evaluate the impact of LARS on gastric (GB) and supragastric belching (SGB) in children with GERD. Methods We performed a prospective, Dutch multicenter cohort study including 25 patients $(12$ males, median age 6 (range 2-18) years) with PPI-resistant GERD who were scheduled for LARS. Twenty-fourhour multichannel intraluminal impedance $\mathrm{pH}$ monitoring (MII-pH monitoring) was performed before and 3 months after fundoplication. Impedance $\mathrm{pH}$ tracings were analyzed for reflux episodes and GBs and SGBS. Key Results LARS reduced acid exposure time from $8.5 \%(6.0-16.2 \%)$ to $0.8 \%(0.2-2.8 \%), p<0.001$. The number of GBs also significantly decreased after LARS (59 [43-77] VS 5 [2-12], $p<0.001)$. The number of air swallows remained unchanged after LARS. SGBS were infrequent before $L A R S$ with no change in the number of SGB observed after the procedure. Postoperative belching symptoms were associated with GBs, not with $S G B S$. Conclusion \& Inferences LARS significantly reduces the number of GBS in children with GERD, 
whereas the number of air swallows remains unchanged. Postoperative symptomatic belching is associated with GBs, but not with SGBs. These findings suggest that LARS does not induce the occurrence of $S G B s$ in children, but longer follow-up is required.

Keywords belching, gas, gastroesophageal reflux disease, laparoscopic fundoplication, pediatrics.

Abbreviations: EGJ, esophagogastric junction; GB, gastric belch; GER, gastroesophageal reflux; GERD, gastroesophageal reflux disease; GSQ, Gastroesophageal Reflux Symptom Questionnaire; LARS, laparoscopic antireflux surgery; MII, multichannel intraluminal impedance; SGB, supragastric belch; TLESR, transient lower esophageal sphincter relaxation.

\section{INTRODUCTION}

The esophagogastric junction (EGJ), composed of the lower esophageal sphincter and crural diaphragm, serves as a physiological barrier that allows the antegrade passage of food and fluids during swallowing, whereas preventing retrograde passage (reflux) of gastric content into the esophagus. ${ }^{1}$ Furthermore, the EGJ mediates gastric belching via transient lower esophageal sphincter relaxation (TLESR). These relaxations are elicited by distention of the gastric fundus and are vital for venting of accumulated intragastric air, ingested with food or during speech. ${ }^{2,3}$ In children with gastroesophageal reflux disease (GERD), the barrier function of the EGJ is impaired and reflux of gastric content into the esophagus is no longer prevented. Most reflux episodes in children occur during these TLESRs. ${ }^{4-6}$

Laparoscopic antireflux surgery (LARS) is a wellestablished treatment option for proton pomp inhibitor (PPI) therapy-resistant GERD in children. ${ }^{7}$ By wrapping the gastric fundus around the lower esophageal sphincter LARS aims to reinforce the barrier function of the EGJ and prevent reflux episodes. In previous studies it has been shown that LARS effectively reduces reflux episodes in adults mainly by a reduction in the number of TLESRs and its association with reflux episodes. ${ }^{8,9}$ However, by reducing the TLESRs, LARS may also impair the physiologic ability to belch and induce postprocedural gas-related symptoms. Previous studies in adults after LARS have demonstrated an impaired belching response during gastric air infusion and a remarkable reduction in the number of postoperative gastric belches (GBs) on 24-h multichannel intraluminal impedance-pH (MII-pH). ${ }^{10-12}$ The inability to vent gastric air post-LARS may lead to gas-related symptoms, such as gas bloating or the inability to belch, which are common complaints after this procedure in adults. ${ }^{13,14}$ Furthermore, 24-h MII-pH tracings also revealed a significant increase in the number of supragastric belches (SGBs). This alternative belching mechanism is behavioral and can be differentiated from GBs as air is sucked into the esophagus and immediately (within a second) expelled again without entering the stomach. ${ }^{15-}$

${ }^{17}$ It was previously hypothesized that the increase in SGBs after LARS is a response to gas bloating, induced by the decrease in GBs and the unaltered rate in air swallows after LARS. The act of supragastric belching, despite no air is vented from the stomach, may alleviate postoperative gas bloating as patients may not be able to discriminate between GBs and SGBs. ${ }^{11}$

Studies on LARS in the pediatric population report the inability to belch in up to $19 \%$ of patients ${ }^{18}$ and gasbloat syndrome in up to $11 \%$ of patients. ${ }^{18-20}$ However, objective data on the effect of LARS on belching and a possible increase in supragastric belching have not been reported. The aim of this study is therefore to objectively evaluate the impact of LARS on belching pattern in children with GERD. We hypothesize that similar to the adult population LARS reduces GBs and in response may induce SGBs.

\section{METHODS}

\section{Study design}

This study contains a post hoc analysis of a prospective multicenter study evaluating the efficacy and safety of laparoscopic antireflux surgery in children in three University Medical Centers in the Netherlands that are specialized in performing fundoplication in children (Wilhelmina children's Hospital, University Medical Center Utrecht [UMCU]; Sophia's Childrens Hospital, Erasmus University Medical Center [Erasmus MC]; and Maastricht University Medical Center [MUMC]). From July 2011 until December 2013, patients diagnosed with PPI therapy-resistant GERD were prospectively included. Patients who underwent previous esophageal or gastric surgery (except previous gastrostomy placement) and those with anatomical abnormalities other than esophageal hiatal hernia were excluded. These patients were evaluated before and 3 months after the surgical procedure.

\section{Patients}

In total 25 children were included in our study. Mean age of the included patients was 6 (range 2-18) years at the time of fundoplication (Table 1$)$. Five children $(80 \%)$ had normal neurodevelopment $(\mathrm{NN})$, whereas impaired neurodevelopment (NI) was seen in five children $(20 \%)$. Underlying diseases causing NI are also shown in Table 1.

\section{Surgical procedure}

Experienced pediatric surgeons performed the laparoscopic fundoplications. In the UMC Utrecht, the anterior, partial 
fundoplication according to Thal ${ }^{21}$ was used to perform fundoplication. In the other two centers (Erasmus MC and Maastricht $\mathrm{UMC}$ ), the posterior, total fundoplication according to Nissen ${ }^{22}$ was performed. Before fundoplication, the distal esophagus was fully mobilized; the distal $3 \mathrm{~cm}$ of the esophagus was repositioned back into the abdomen. Furthermore, in the UMCU and EMC, both vagal nerves were identified, and a cruroplasty was routinely performed. Thereafter, the fundoplication was constructed. The Thal fundoplication was performed by plication of the fundus of the stomach over $270{ }^{\circ} \mathrm{C}$ against the distal anterior intra-abdominal part of the esophagus and the diaphragmatic crus. ${ }^{23}$ A floppy Nissen was constructed with one of the sutures of the $360{ }^{\circ} \mathrm{C}$ posterior wrap incorporated into the esophageal wall. $^{22}$

\section{Ambulatory 24-h multichannel intraluminal impedance $\mathrm{pH}$ monitoring}

Ambulatory 24-h MII-pH testing was conducted after at least 3 days cessation of all medications that affect gastrointestinal motility and/or acid secretion. Measurements were performed using an age-adjusted combined $\mathrm{pH}$-impedance catheter assembly that consisted of six impedance segments and one ISFET $\mathrm{pH}$ electrode (Unisensor AG, Attikon, Switzerland). Impedance and $\mathrm{pH}$ signals were stored on a digital datalogger (Ohmega, Medical Measurement Systems, Enschede, The Netherlands), using a sampling frequency of $50 \mathrm{~Hz}$. Patients and/or their parents were instructed to continue their regular diet, to report GERD symptoms, and to keep a diary of their consumptions and body position (supine or upright) during the measurement.

\section{Reflux-specific questionnaires}

To assess belching symptoms patients and/or their parents were asked to fill out the validated age-adjusted Gastroesophageal Reflux Symptom Questionnaire (GSQ) before and 3 months after LARS. ${ }^{24}$ Patients and/or their parents monitored belching symptoms during the week before and including the day of MII-pH monitoring. The time interval before surgery ranged between 1 week and 2 months. Postoperative symptom assessment was performed 3 months after surgery. Belching symptoms were scored for frequency and severity on a score ranging from 1 (none) to 7 (most severe). Symptoms were defined as: no symptoms (no symptoms reported); mild (mild symptoms, weekly); moderate (mild symptoms daily or severe symptoms weekly); and severe (severe symptoms daily).

Table 1 Baseline characteristics

\begin{tabular}{ll}
\hline & Median (IQR) or $n(\%)$ \\
\hline Age at time of operation (years) & $6.0(3.0-11.0)$ \\
Duration of hospital admission (days) & $3.0(2.0-4.5)$ \\
Male gender & $12(48.0)$ \\
Impaired neurodevelopment & $5(20.0)$ \\
- Charge syndrome & \\
- Mitochondrial complex II deficiency & \\
- Post-hypoxic encephalopathy & \\
- Congenital rubella infection & \\
- Impaired neurodevelopment of unknown & \\
$\quad$ origin with autistic behavior & \\
\hline
\end{tabular}

IQR, interquartile range.

\section{Data analysis}

All 24-h MII-pH tracings were manually analyzed for the number of GBs and SGBs according to previously described criteria. ${ }^{15}$ In short, GBs were defined as a rapid retrograde rise in impedance of $>3000 \Omega$ in at least two consecutive impedance channels reaching the most proximal impedance channel. SGBs were defined by rapid, non-peristaltic, antegrade entry of air into the esophagus (increase in baseline impedance of $>1000 \Omega$ ), directly followed by quick expulsion of air (Fig. 1). The 24-h MII-pH tracings were further evaluated for air swallows, acid exposure time, and the number and content of liquid-containing reflux episodes according to international accepted definitions. ${ }^{25}$ Pathologic acid exposure time was defined as $\mathrm{pH}<4$ during $>6.0 \%$ of time during 24 -h monitoring. ${ }^{26,27}$

\section{Ethical approval and trial registration}

This study was registered in the Dutch national trial registry (www.trialregister.nl; Identifier: 2934). Ethical approval for this prospective multicenter study was obtained from the UMC Utrecht Ethics Committee and local approval was obtained by the remaining participating centers. Prior to initiating any trialrelated study procedure, informed consent from the patients' parents was obtained.

\section{Statistical analysis}

Continuous parametric variables were expressed as mean \pm standard error of the mean (SEM). Non-parametric variables were expressed as median, with interquartile ranges (IQR). Exploratory subgroups analyses for all outcome measures were performed comparing type of fundoplication and neurodevelopment and using a paired sample T-test for continuous parametric outcomes and the Wilcoxon signed rank test for non-parametric outcomes. Correlations between different parameters were calculated using the Pearson correlation coefficient or Spearman's rank correlation coefficient as appropriate. Linear regression analysis was performed to identify possible determinants of the effect of LARS on belching. Determinants of interest included: age, type of fundoplication, neurologic development, and the number of air swallows. A $p$-value below 0.05 was considered statistically significant. All statistical analyses were performed using commercially available computer software (IBM SPSS Statistics for Windows, Version 22; IBM Corp., Armonk, NY, USA).

\section{RESULTS}

In total 18 Thal and 7 Nissen fundoplications were performed. In all patients fundoplication could be completed by laparoscopy. Perioperative or postoperative complications were not observed.

Preoperative 24-h pH-MII tracings were completed in all 25 patients. After surgery, 24-h MII-pH monitoring was successfully completed in 23 of 25 children. In two patients postoperative tracings could not be performed due to refusal by the caregivers. The caregivers of one of these two patients also did not fill out the postoperative symptom questionnaires. Both patients were left out of the analysis. 

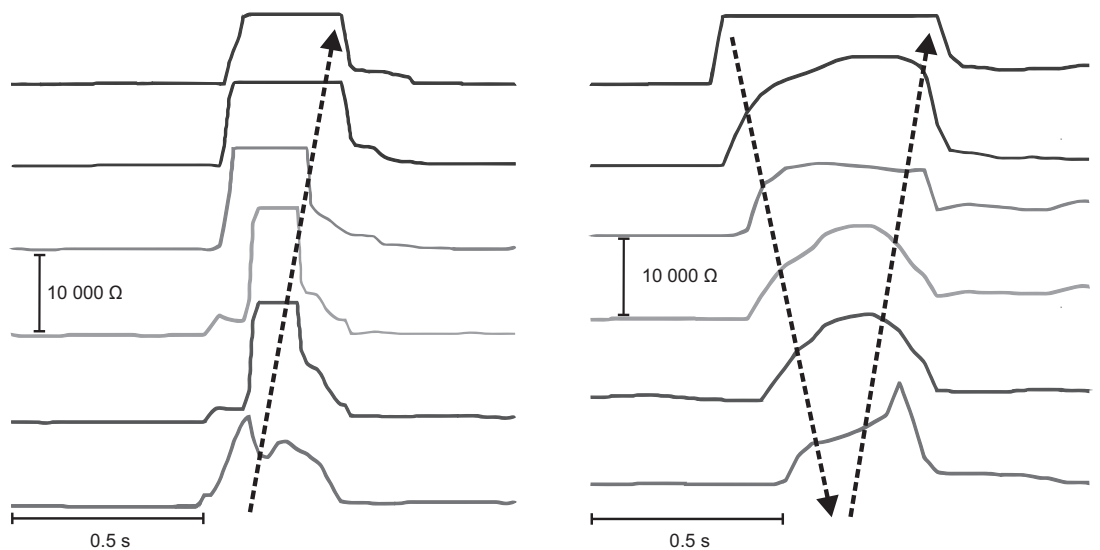

Figure 1 Examples of gastric belch (left panel) and supragastric belch (right panel) on 24-h MII-pH tracing. Arrows indicate air flow.

\section{Reflux control}

LARS successfully reduced the number of reflux episodes and thereby decreased esophageal acid exposure time (Table 2). Both pure liquid and mixed (liquid and gas) reflux episodes were significantly reduced after LARS (Table 2). In one patient with retching based on impaired neurodevelopment a redofundoplication was indicated because of severe recurrent reflux (pathological reflux on 24-h pH monitoring and severe reflux symptoms) caused by hiatal herniation.

\section{Belching}

Before LARS, GBs were frequently observed in all pediatric GERD patients. Approximately half of these GBS were directly followed by or occurred during a reflux episode (Table 3). The total number of GBs was significantly reduced after LARS (59 [43-77] VS 5 [2-12], $p<0.001$ ) (Table 3). In three patients GBs were no longer identified on postoperative 24-h pH-MII tracings (Fig. 2). SGBs occurred infrequently both before and after LARS and no change in the number of SGBs was found (Table 3). However, in one child the number of SGBs increased up to over 170 SGBs per day (Fig. 3). Nonetheless, this patient had no complaints of belching after LARS.

Symptomatic belching significantly decreased after LARS (Table 4). Postoperative belching symptoms were correlated with the number of GBs $(r: 0.53$, $p<0.01$ ), not with SGBs (r: 0.35, NS). Furthermore, patients who reported moderate-to-severe belching symptoms after the procedure showed a trend toward more GBs when compared to patients without or with only mild belching symptoms (resp. 9 [4-26] VS 4 [0-7], $p=0.06)$, but a similar low number of SGBs (resp. 2 [04] VS 1 [0-4], NS).

\section{Impact of type of fundoplication and neurodevelopment on belching}

To explore the possible differences between types of fundoplication used in this study, patients were divided into two groups: one group that had undergone a partial (Thal) fundoplication $(n=17)$ and one group that had undergone a complete (Nissen) fundoplication $(n=6)$. Gastric belches decreased significantly after both partial (from 66 [45-87) to 5 [2-12], $p<0.001$ ) and complete (from 44 [37-57] to 7 [4-15], $p=0.03$ ) fundoplication. The reduction in GBs after partial fundoplication $(-55[-74,-41])$ and complete fundoplication $(-36[-51,-25])$ was not significantly different $(p=0.14)$. Also the number of postoperative GBs (partial: 5 [2-12] vs complete: 7 [4-15], $p=0.18$ ), SGBs (partial: $2[0-5]$ vs complete: $3[0-4], p=0.37)$, and severity of postoperative belching symptoms (partial: 2 [0-49] VS 5 [0-11], $p=0.84$ ) were not different between partial and complete fundoplication.

Table 2 Reflux parameters before and after LARS on MII-PH monitoring

\begin{tabular}{lccr}
\hline & Preoperative & Postoperative & $p$-value \\
\hline $\begin{array}{l}\text { Duration (hours } \\
\text { [median, range] }\end{array}$ & $22.5(20.1-23.9)$ & $21.8(16.1-24.0)$ & \\
$\begin{array}{l}\text { Percentage of time } \\
\text { in supine }\end{array}$ & $52.4 \pm 2.6$ & $49.0 \pm 1.7$ & \\
position (\% \pm SEM) & & & \\
Acid exposure & $8.5(6.0-16.2)$ & $0.8(0.2-2.8)$ & $<0.001$ \\
time (\%, IQR) & & & \\
Upright (\%, IQR) & $12.1(4.8-19.2)$ & $1.8(0.5-5.9)$ & 0.001 \\
Supine (\%, IQR) & $7.1(0.9-15.3)$ & $0(0-0)$ & $<0.001$ \\
Total GER episodes & $92(66-139)$ & $14(11-22)$ & $<0.001$ \\
(n, IQR) & & & $<0.001$ \\
Liquid reflux & $51(40-93)$ & $10(5-19)$ & $<0.001$ \\
Mixed reflux & $38(21-50)$ & $3(1-8)$ & $<$ \\
\hline
\end{tabular}

IQR, interquartile range; LARS, laparoscopic antireflux surgery. 
Table 3 Number of GB and the association with reflux episodes, supragastric belches, and air swallows during 24-h MII-pH monitoring

\begin{tabular}{lccl}
\hline & Preoperative & Postoperative & $p$-value \\
\hline Gastric belches (total) & $59(43-77)$ & $5(2-12)$ & $<0.001$ \\
GB alone & $30(14-53)$ & $4(1-8)$ & $<0.001$ \\
GB during reflux & $5(2-9)$ & $0(0-1)$ & $<0.001$ \\
GB prereflux & $19(10-26)$ & $1(0-2)$ & $<0.001$ \\
Supragastric belches & $2(0-7)$ & $2(0-4)$ & NS \\
Air swallows & $39(29-65)$ & $43(28-83)$ & NS \\
\hline
\end{tabular}

GB, gastric belch; MII-pH, multichannel intraluminal impedance-pH.

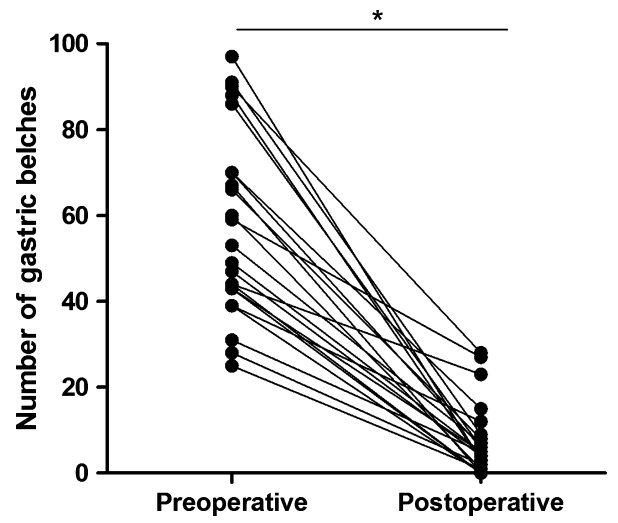

Figure 2 Pre- and postoperative number of gastric belches for each individual patient on $24-\mathrm{h}$ MII-pH monitoring. ${ }^{*} p<0.001$.

Patients with impaired neurodevelopment (NI; $n=5)$ and normal neurodevelopment $(\mathrm{NN} ; n=18)$ were also compared with respect to pre- and postoperative GBs and SGBs. No significant differences were observed between the two groups with respect to preoperative GBs (NI: 70 [54-94] vs NN: 50 [42-72]), preoperative SGBs (NI: 7 [6-8] vs NN: 1 [0-4], postoperative GBs (NI: 6 [4-28] vs NN: 5 [1-10]), and postoperative SGBs (NI: 2 [2-3] vs NN: 2 [0-5]).

\section{Predictors for the effect of LARS on belching}

A linear regression analysis was performed to explore determinants of the effect of LARS on gastric and supragastric belching. No significant predictor (including type of fundoplication and neurodevelopment) was identified predicting the effect of LARS on the number of gastric and supragastric belching (data not shown). Linear regression analysis, however, did identify the number of preoperative air swallows as a determinant positively influencing the number of preoperative gastric belching ( $\beta$ : $0.24 ; 95 \%$ CI: 0.04-0.44, $p=0.03$ ). After fundoplication, however, the number of air swallows was not associated with the number of postoperative belches. Nonetheless, postoperative

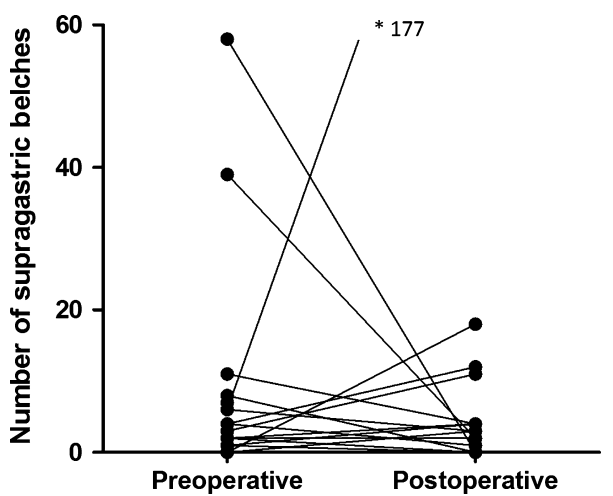

Figure 3 Pre- and postoperative number of supragastric belches for each individual patient on 24-h MII-pH monitoring.

Table 4 Assessment of symptomatic belching: number of patients and severity of belching symptoms before and after LARS

\begin{tabular}{llll}
\hline $\begin{array}{l}\text { Belching } \\
\text { symptoms }\end{array}$ & $\begin{array}{l}\text { Preoperative } \\
(n ; \%)\end{array}$ & $\begin{array}{l}\text { Postoperative } \\
(n ; \%)\end{array}$ & $p$-value \\
\hline None & $5(21)$ & $9(37)$ & 0.007 \\
Mild & $3(13)$ & $7(29)$ & \\
Moderate & $8(33)$ & $5(21)$ & \\
Severe & $8(33)$ & $3(13)$ & \\
\hline
\end{tabular}

LARS, laparoscopic antireflux surgery.

gastric belches were associated with postoperative belching symptoms ( $\beta$ : 0.43 ; $95 \%$ CI: $0.06-0.80$, $p=0.04$ ), as previously established. All other determinants of interest did not show any significant effect on the effect of LARS on belching and the total number of belches before and after LARS.

\section{DISCUSSION}

This is the first study that explores the effect of LARS on belching in pediatric GERD patients. The main findings were that belching symptoms and number of GBs significantly decreased after LARS, but LARS did not affect the number SGBs.

In previous studies, it has been reported that $19 \%$ of pediatric patients are unable to belch and up to $11 \%$ suffer from gas-bloat syndrome after LARS. ${ }^{18-20}$ Although it is generally accepted that these symptoms arise from accumulation of intragastric air as a consequence of an inability to belch, the substantial reduction in the number of GBs after LARS as observed in this study provides objective data supporting this hypothesis on the pathogenesis of these postoperative symptoms in children. These results are in line with studies in adults, in whom a similar reduction in GBs was observed after LARS. ${ }^{10-12}$ 
The mechanism through which LARS leads to a reduction in GBs was previously thought to be due to the reduction in TLESRs after LARS. ${ }^{10}$ GBs are mediated mainly by TLESRs, which allow gastric air to escape from the stomach into the esophagus. Consequently, by relaxation of the upper esophageal sphincter air passes into the pharynx and mouth. ${ }^{2,3,28}$ Previously, several studies have shown that the number of TLESRs is significantly reduced after LARS. ${ }^{8,9}$ This reduction in TLESRs correlated with impaired gastric belching and consequently led to gas-related complications after LARS. ${ }^{10}$ In the current protocol, assessment of the effect of LARS on TLESRs and their association with belching was not included. We, however, speculate that similar to adults, the reduction in TLESRs is mainly responsible for the decrease in GBs. The reduction in GBs cannot be explained by a change in air swallowing, as the number of air swallows after LARS remained unchanged. This is similar to data in adult studies on belching patterns after LARS. ${ }^{11,12}$ The number of air swallows was, however, lower in pediatric GERD patients compared to the adult GERD patients, although comparable to the rate of air swallows previously reported in healthy children. $^{29}$

Supragastric belching was not frequently observed and no shift in belching patterns from gastric to supragastric belching occurred in pediatric GERD patients. This is in contrast to findings from two studies in adults, in which the number of SGBs increased and caused a higher number of postoperative belching symptoms. ${ }^{11,12}$ In contrast, in children postoperative belching symptoms decreased and remaining belching symptoms after LARS correlated with GBs but not with SGBs. The number of SGBs in the current population was relatively low compared to findings from adult studies. ${ }^{11,12}$ The current results suggest that supragastric belching plays no significant role in pediatric GERD patients. However, further studies are needed to support and confirm this statement, as up to now no studies have been performed to assess the relationship between supragastric belching and GERD specifically and more in general, gas-related complaints and objectively identified abnormalities such as supragastric belching or aerophagia in children.

Nevertheless, excessive supragastric belching after LARS may occur, as we observed in a single patient. This specific child exhibited autistic behavior with impaired neurodevelopment that may have (at least partly) contributed to this abnormal belching pattern after LARS.
In this study, two types (partial and complete) of fundoplication were performed that may have a different effect on belching. In a study on belching after LARS in adult GERD patients, with aerophagia as comorbidity, the gas-related symptomatic outcome of partial fundoplication was superior to complete fundoplication. ${ }^{30}$ This finding is in line with several studies and meta-analyses showing favorable outcomes of partial fundoplication compared to complete fundoplication with regard to gas-related complications of LARS. ${ }^{10,12-14}$ We performed an exploratory comparison between the effects of the different types of fundoplication, showing no apparent advantage comparing complete-to-partial fundoplication in maintaining the ability to belch. However, it has to be noted that this study was not powered to answer this question. Therefore, future studies, preferably in the form of a randomized controlled trial, should be performed to give more conclusive answers.

Another issue that needs to be considered regarding the conclusions of this study is that a 3-month follow-up is relatively short as impaired belching may improve over a longer period of time. Allal et al. previously described that after 10 months gas bloating had resolved completely, but three patients with severe complaints required placement of a gastric tube in their study. Moreover, other studies have reported gas-related complications in a substantial proportion of children several years after LARS. ${ }^{18,31}$ Lastly, although this study provides objective evidence for intragastric gas accumulation, we have no data to support the assumption that intragastric gas accumulation leads to gas-bloat symptoms and hyperflatulence as frequently reported after fundoplication.

In conclusion, gastric belching is significantly reduced after LARS, whereas the number of air swallows remains unchanged. This is likely to explain frequently reported postoperative gas-related complications (gas bloat and the inability to belch) of LARS in children. Supragastric belches occurred infrequently and the number of SGBs was not altered after LARS. Additional studies on the evolution of gas-related complications during long-term follow-up and possible differences between commonly used surgical techniques are needed.

\section{FUNDING}

F.A. Mauritz is supported by a Wilhelmina Children's Hospital Grant and the NutsOhra. No other sources of support were used. 


\section{DISCLOSURE}

No competing interests declared.

\section{AUTHOR CONTRIBUTIONS}

NFR: study design, acquisition of data, analysis and interpretation of data, drafting of the manuscript; FAM: study design, acquisition of data, analysis and interpretation of data, drafting of the manuscript; EVH: acquisition of data, critical revision of the manuscript; CES: acquisition of data, critical revision of the manuscript; PDS: critical revision of the manuscript; $\mathrm{RH}$ : critical revision of the manuscript; DCZ: study design, acquisition of data, and critical revision of the manuscript; AAM: critical revision of the manuscript; JMC: critical revision of the manuscript; MHL: study design, acquisition of data, analysis and interpretation of data, critical revision of the manuscript.

\section{REFERENCES}

1 Mittal RK, Balaban DH. The esophagogastric junction. N Engl I Med 1997; 336: 924-32

2 Wyman JB, Dent J, Heddle R, Dodds WJ, Toouli J, Downton J. Control of belching by the lower oesophageal sphincter. Gut 1990; 31: 639-46.

3 Kessing BF, Conchillo JM, Bredenoord AJ, Smout AJ, Masclee AA. Review article: the clinical relevance of transient lower oesophageal sphincter relaxations in gastro-oesophageal reflux disease. Aliment Pharmacol Ther 2011; 33: 650-61.

4 Werlin SL, Dodds WJ, Hogan WJ, Arndorfer RC. Mechanisms of gastroesophageal reflux in children. I Pediatr 1980; 97: 244-9.

5 Cucchiara S, Bortolotti M, Minella R, Auricchio S. Fasting and postprandial mechanisms of gastroesophageal reflux in children with gastroesophageal reflux disease. Dig Dis Sci 1993; 38: 86-92.

6 Kawahara H, Dent J, Davidson G. Mechanisms responsible for gastroesophageal reflux in children. Gastroenterology 1997; 113: 399-408.

7 Fuchs KH, Babic B, Breithaupt W, Dallemagne B, Fingerhut A, Furnee E, Granderath F, Horvath $\mathrm{P}$ et al. EAES recommendations for the management of gastroesophageal reflux disease. Surg Endosc 2014; 28: 1753-73.

8 Lindeboom MA, Ringers J, Straathof JW, van Rijn PJ, Neijenhuis P, Masclee AA. Effect of laparoscopic partial fundoplication on reflux mechanisms. Am I Gastroenterol 2003; 98: 29-34.

9 Bredenoord AJ, Draaisma WA, Weusten BL, Gooszen HG, Smout AJ. Mechanisms of acid, weakly acidic and gas reflux after anti-reflux surgery. Gut 2008; 57: 161-6.
10 Straathof JW, Ringers J, Lamers CB, Masclee AA. Provocation of transient lower esophageal sphincter relaxations by gastric distension with air. Am I Gastroenterol 2001; 96: 2317-23.

11 Broeders JA, Bredenoord AJ, Hazebroek EJ, Broeders IA, Gooszen HG, Smout AJ. Effects of anti-reflux surgery on weakly acidic reflux and belching. Gut 2011; 60: 435-41.

12 Broeders JA, Bredenoord AJ, Hazebroek EJ, Broeders IA, Gooszen HG, Smout AJ. Reflux and belching after 270 degree versus 360 degree laparoscopic posterior fundoplication. Ann Surg 2012; 255: 59-65.

13 Broeders JA, Mauritz FA, Ahmed Ali U, Draaisma WA, Ruurda JP, Gooszen HG, Smout AJ, Broeders IA et al. Systematic review and meta-analysis of laparoscopic Nissen (posterior total) versus Toupet (posterior partial) fundoplication for gastro-oesophageal reflux disease. Br I Surg 2010; 97: 1318-30.

14 Broeders JA, Roks DJ, Ahmed Ali U, Watson DI, Baigrie RJ, Cao Z, Hartmann J, Maddern GJ. Laparoscopic anterior 180-degree versus nissen fundoplication for gastroesophageal reflux disease: systematic review and meta-analysis of randomized clinical trials. Ann Surg 2013; 257: 850-9.

15 Bredenoord AJ, Weusten BL, Sifrim D, Timmer R, Smout AJ. Aerophagia, gastric, and supragastric belching: a study using intraluminal electrical impedance monitoring. Gut 2004; 53: 1561-5.

16 Kessing BF, Bredenoord AJ, Smout AJ. The pathophysiology, diagnosis and treatment of excessive belching symptoms. Am I Gastroenterol 2014; 109: 1196-203; (Quiz) 1204.

17 Kessing BF, Bredenoord AJ, Smout AJ. Mechanisms of gastric and supragastric belching: a study using concurrent high-resolution manometry and impedance monitoring. Neurogastroenterol Motil 2012; 24: e5739

18 Kwiecien J, Dzielicki J, Korlacki W, Ciekalski J, Zabka A, Krzywicka A, Kasner J, Pieniazek W et al. Long-term effects of laparoscopic antireflux surgery in children with gastroesophageal reflux disease. Pediatria Wspolczesna 2004; 6: 249-53.

19 Allal H, Captier G, Lopez M, Forgues D, Galifer RB. Evaluation of 142 consecutive laparoscopic fundoplications in children: effects of the learning curve and technical choice. I Pediatr Surg 2001; 36: 921-6.

20 Kazerooni NL, VanCamp J, Hirschl RB, Drongowski RA, Coran AG. Fundoplication in 160 children under 2 years of age. I Pediatr Surg 1994; 29: 677-81.

21 Thal AP. A unified approach to surgical problems of the esophagogastric junction. Ann Surg 1968; 168: 542-50.

22 Nissen R. A simple operation for control of reflux esophagitis. Schweiz Med Wochenschr 1956; 86: 590-2.

23 Mauritz FA, van Herwaarden-Lindeboom MY, Zwaveling S, Houwen RH, Siersema PD, van der Zee DC. Laparoscopic Thal fundoplication in children: a prospective 10- to 15-year follow-up study. Ann Surg 2014; 259: 388-93.

24 Deal L, Gold BD, Gremse DA, Winter HS, Peters SB, Fraga PD, Mack ME, Gaylord SM et al. Age-specific questionnaires distinguish GERD symptom frequency and severity in infants and young children: development and initial validation. I Pediatr Gastroenterol Nutr 2005; 41: 178-85.

25 Sifrim D, Holloway R, Silny J, Xin Z, Tack J, Lerut A, Janssens J. Acid, 
nonacid, and gas reflux in patients with gastroesophageal reflux disease during ambulatory 24-hour $\mathrm{pH}$-impedance recordings. Gastroenterology 2001; 120: 1588-98.

26 Smout AJ, Breedijk M, van der Zouw C, Akkermans LM. Physiological gastroesophageal reflux and esophageal motor activity studied with a new system for 24-hour recording and automated analysis. Dig Dis Sci 1989; 34: 372-8.

27 Richter JE, Bradley LA, DeMeester TR, Wu WC. Normal 24-hr ambulatory esophageal $\mathrm{pH}$ values. Influence of study center, $\mathrm{pH}$ electrode, age, and gender. Dig Dis Sci 1992; 37: 849-56.

28 Dent J, Holloway RH, Toouli J, Dodds WJ. Mechanisms of lower oesophageal sphincter incompetence in patients with symptomatic gastrooesophageal reflux. Gut 1988; 29: 1020 8.

29 Halb C, Pomerleau M, Faure C. Multichannel intraesophageal impedance pattern of children with aerophagia. Neurogastroenterol Motil 2014; 26: 1010-4.

30 Granderath FA, Kamolz T, Granderath UM, Pointner R. Gas-related symptoms after laparoscopic 360 degrees Nissen or 270 degrees Toupet fundoplication in gastrooesophageal reflux disease patients with aerophagia as comorbidity. Dig Liver Dis 2007; 39: 312-8.

31 Pacilli $M$, Eaton S, McHoney M, Kiely EM, Drake DP, Curry JI, Lindley KJ, Pierro A. Four year follow-up of a randomised controlled trial comparing open and laparoscopic Nissen fundoplication in children. Arch Dis Child 2014; 99: 516-21. 